\title{
Effect of Vortex-Wake Interaction on Vortex-Rotor Interference and on Rotor Trim
}

\author{
Berend G. van der Wall ${ }^{(凶)}$ (D) \\ German Aerospace Center (DLR), Institute of Flight Systems (FT), Lilienthalplatz 7, \\ 38108 Braunschweig, Germany \\ berend. vanderwalladlr. de
}

\begin{abstract}
The flight of a helicopter within the wake of a preceding fixed-wing aircraft, for example during air-to-air refueling, is accompanied by vortex-rotor interactions. The full mutuality of vortex and rotor wake interactions requires a free-wake solution that is applied at different advance ratios in this paper and compared to existing simplified models. The results indicate that the simplified models are valid for higher advance ratios whereas very small advance ratios require the use of a free-wake approach.
\end{abstract}

\section{Introduction}

Helicopters can encounter wing tip vortices or those generated by deflected flaps from preceding fixed-wing aircraft, which becomes important during air-to-air refueling of helicopters: when approaching to the refueling position, while refueling, and when departing from it. Then the heavy tanker aircraft flies close to its minimum speed with deflected flaps, while the rotorcraft flies close to its maximum speed. Figure 1(a) shows this type of interaction and the fundamental kind of the resulting disturbance within the rotor disk is illustrated in Fig. 1 (b).

Aerodynamic interference is mentioned in most textbooks as an important subject that drives the design of components of a helicopter [1,2]. Its analytic treatment is often very complicated and left to computational fluids dynamics (CFD) codes, for example the fuselage-rotor, rotor-tail rotor, rotor-empennage and other interaction problems. A good overview of the multitude and mutuality of interactions amongst helicopter components and with the outer environment is given in [3]. The problem of an external vortex interacting with the helicopter main rotor and its wake has been investigated since the 1970s regarding the helicopter flight into a wide body aircraft wake with respect to flight dynamic response and handling qualities of the rotorcraft [4-6]. It was concluded that the heavier (larger) the helicopter, the less impact the aircraft wake has on the helicopter. Recently helicopter flight within offshore wind parks was the subject of a GARTEUR research activity [7]. The work encompassed a survey of experimental and numerical studies about the wind turbine wake characteristics in the near and far field behind them; computer simulations to assess stability and handling qualities of a helicopter flying across wind turbine wakes; and piloted simulations using ground-based simulators. 


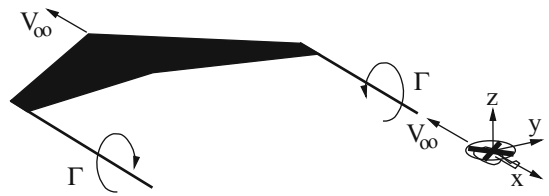

(a) Helicopter in fixed-wing wake

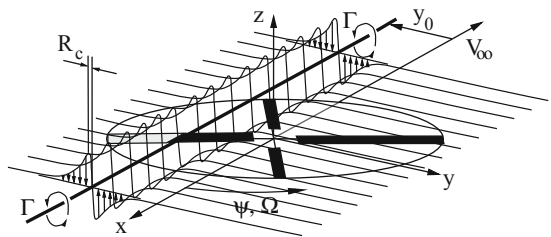

(b) Helicopter rotor with a line vortex

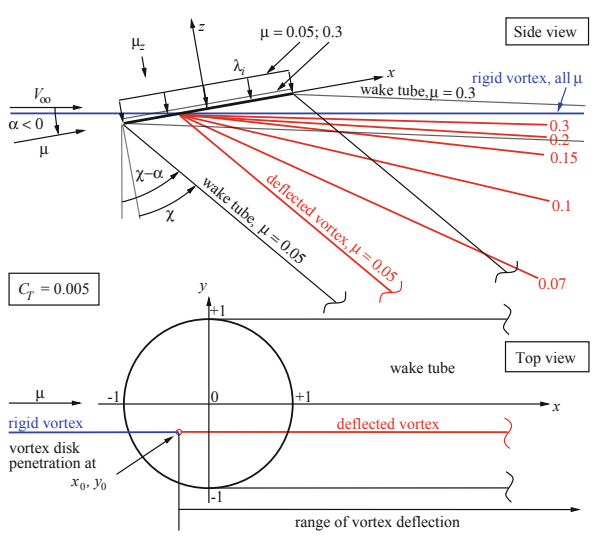

(c) Rigid straight-line and deflected vortex models for different advance ratios

Fig. 1. Illustration of vortex - rotor interaction.

In the past, most numerical investigations considered the vortex-rotor interaction by assuming the vortex as space-fixed (rigid straight-line vortex model) and only considering its induced velocities at the rotor blades; while vortex-wake mutual interactions were ignored. This article introduces a unidirectional interaction by deflecting the vortex when penetrating the rotor disk (deflected vortex model) and the full mutuality of interaction by application of a free-wake method to both the external vortex and the rotor wake. The strongest simplification is assuming a rigid straight-line vortex without any vortex-wake interaction at all as illustrated in Fig. 1 (c) by the blue line. This is the most conservative approach, leading to the strongest vortex impact on rotor trim. For a vortex lying planar in the rotor disk an analytical solution was derived for the rotor controls needed for disturbance rejection [8], or the perturbations developing in rotor blade flapping [9], or the thrust and power disturbances that occur when no controls are applied [10]. A vortex with its axis perpendicular to the rotor plane was investigated in [11].

A simplified unidirectional interaction where the vortex is deflected into the rotor wake slipstream, but without impact on the slipstream itself, was recently investigated in [12]. This is exemplified in Fig. 1 (c) for a wide range of advance ratios $\mu$ for a constant thrust coefficient $C_{T}$ by the red lines, indicating the deflected part of the vortex after reaching the rotor disk. Because of diminishing induced inflow ratio $\lambda_{i}$ at high speed the wake skew angle $\chi$ and with it the vortex deflection becomes very small and the vortex remains close to the rotor disk. In [13] CFD was applied to the problem for a few cases and results were compared to rigid vortex assumptions. The full mutuality of interactions between vortex and rotor wake showed a significant reduction of vortex-rotor interaction impact on trim.

An intermediate level of fidelity can be obtained by a free-wake simulation where both the vortex and the rotor wake interact with each other. This is the approach used in this paper and the obtained results will be compared to the other methods. All the simplified approaches - including free-wake methods - are typical for comprehensive rotor 
codes, while CFD codes inherently include all interactional effects, but at a computational effort some orders of magnitude larger.

\section{Technical Approach}

Rigid Straight-Line Vortex Model. A Vatistas' type of vortex swirl model as given in Eq. (1) is used, which computes the normal vortex-induced velocity at any rotor blade element $(x, y)$ or radial and azimuth position $(r, \psi)$ for a vortex axis lateral position $y_{0}$ with respect to the rotor center. $\Gamma_{V}$ is the dimensional and $\lambda_{V 0}$ the nondimensional vortex circulation strength, referenced to the interacting rotor's tip speed $\Omega R$ and radius $R$. $\lambda_{V 0}$ is a linear measure for the impact on the trim of the encountering rotor, expressed by the perturbations of the collective and cyclic control angles $\Delta \Theta_{0}, \Delta \Theta_{\mathrm{S}}, \Delta \Theta_{\mathrm{C}}$ needed to mitigate the vortex effect on trim. A nonlinear measure is the nondimensional vortex core radius $r_{c}=R_{c} / R$. The wing vortex core radius $R_{c}$ will be large compared to the rotor blade chord length $c$ [10]. $r_{c}$ is kept almost constant for all computations.

$$
\lambda_{V}=\frac{\Gamma_{V}}{2 \pi \Omega R^{2}} \frac{y-y_{0}}{\left(y-y_{0}\right)^{2}+r_{c}^{2}}=\lambda_{V 0} \frac{r \sin \psi-y_{0}}{\left(r \sin \psi-y_{0}\right)^{2}+r_{c}^{2}}
$$

Deflected Vortex Model. This model [12] makes use of momentum theory as outlined in $[1,2]$ to compute the wake skew angle $\chi$ as a function of the rotor thrust coefficient $C_{T}$, the advance ratio $\mu$ and the inclination angle $\alpha$ of the rotor's tip path plane. The induced inflow ratio $\lambda_{i}$ needs to be solved from the quartic equation given in Eq. (2), where $\lambda_{h}$ is the induced inflow ratio in hover. From the point where the vortex reaches the tip path plane at $\left(x_{0}, y_{0}\right)$, see Fig. 1 (c), the vortex gets deflected by the wake skew angle $\chi$. For the majority of computations $x_{0}=0$ is used throughout in the paper because this allows for the widest range of vortex lateral positions inside the rotor disk $-1 \leq y_{0} \leq 1$, see Fig. 1 (c), top view, where for a range of advance ratios the deflection angles are indicated by the red lines.

$$
\begin{aligned}
& 0=\lambda_{i}^{4}+2 \mu_{z} \lambda_{i}^{3}+\mu^{2} \lambda_{i}^{2}-\lambda_{h}^{4} ; \quad \mu=V_{\infty} \cos \alpha /(\Omega R) ; \quad \mu_{z}=-\mu \tan \alpha \\
& \chi=\arctan \left[\mu /\left(\lambda_{i}+\mu_{z}\right)\right] ; \quad \lambda_{h}=\sqrt{C_{T} / 2}
\end{aligned}
$$

Free-Wake Solution. The free-wake code applied here is based on [16], but the classical vortex lattice method has been replaced by continuous bilinear vorticity distributions for the trailed and shed vorticity in the wake elements, who are combined with a "thickness" representing a core radius. This avoids adaptive grid refinement when evaluation points get close to wake panels. A grid coarsening for points far away from the wake panels is applied to accelerate the computation. The external vortex is generated by a fixed wing located one radius upstream of the rotor. Its circulation strength is computed by the angle of attack and lift curve slope of the wing. In order to have just one tip vortex interacting with the rotor, the wing is oriented vertically with its upper tip just above the rotor hub center and its lower tip a wing span below. This way the concentrated vorticity in the vicinity of the wing tip enters the rotor located downstream of the wing and mixes with the rotor wake, while the other wing tip vortex remains sufficiently away below the rotor wake. The only purpose of this wing is to act as a vortex generator. 
Rotor Simulation Tools. DLR's high resolution comprehensive rotor simulation code S4 [15] was applied to the Bo105 main rotor and HOST - the Helicopter Overall Simulation Tool of Airbus Helicopters [14] - was applied to a Bo105 helicopter fixed in space and subjected to an aircraft wing tip vortex [10]. S4 computes the dynamic response of elastic rotor blades with flap, lag and torsion degrees of freedom. The induced velocities caused by the rotor thrust are represented by the Mangler/Squire model and the resulting induced inflow distributions depend on $C_{T}, \mu, \alpha$. Unsteady aerodynamic modeling of blade element forces and pitching moment are incorporated. For the free-wake solution DLR's VAST (Versatile Aeromechanic Simulation Tool) is used with an articulated rigid blade model of the Bo105 main rotor. The model includes flapping and lead-lag motion with equivalent hinge offset and springs to match rotating natural frequencies of first flap and lead-lag modes. No torsion is taken into account. Linear incompressible aerodynamics are applied to the rotor blade elements and a free-wake is used for both the rotor wake and the wake of the fixed wing located upstream of the rotor. VAST is under development and no publications about it are available.

Rotor, Vortex and Wing. The Bo105 main rotor blades with $R=4.91 \mathrm{~m}$ are discretized into 10 blade elements in VAST and 20 within S4 with progressively smaller elements towards the blade tip in order to better resolve the increasing dynamic pressure along span. The external vortex' core radii, referenced to $R$, are $r_{c}=0.081$ in VAST and 0.1 in HOST and S4, thus the results using VAST with its free-wake may lead to larger control angles than those of S4 with larger core radius and finer blade resolution. A lift curve slope of $C_{l \alpha}=6.9$ is used in VAST and a quadratic drag coefficient polar is employed. While the analytical results are based on $C_{l \alpha}=2 \pi$, the $\mathrm{S} 4$ uses airfoil polars including compressibility. An azimuthal resolution of $3.6 \mathrm{deg}$ is used in VAST, while the $\mathrm{S} 4$ code was run with $2 \mathrm{deg}$ resolution. The rotor shaft angle of attack is prescribed. The wing has a span of $5 \mathrm{~m}$ and an aspect ratio of 5 , discretized into 10 equally spaced blade elements. The core radius in the free-wake code was set to $R_{c}=0.4 \mathrm{~m}$.

Rotor Trim and External Vortex Strength. First, a rotor trim without an external vortex and thus with undisturbed air at the rotor is performed for a range of advance ratios from $\mu=0$ (hover) to 0.4 representing high speed flight. The rotor thrust is prescribed to $24200 \mathrm{~N}\left(C_{T}=0.0055\right)$ and the hub moments to $0 \mathrm{Nm}$. These are the "Baseline" cases. The majority of VAST computations is limited to four selected advance ratios $\mu=0.1,0.2,0.3,0.4$ and the vortex or wing is inserted into the flow with its lateral position relative to the rotor hub center varied in the range $-2 \leq y_{0} \leq 2$ at 11 positions. At any of these a retrim of the rotor was performed and the difference in control angles relative to the baseline trim is evaluated. One computation with VAST is performed at $\mu=0.05$ with $y_{0}=0$, but obtaining a converged trim solution at this low advance ratio takes much more time than at higher $\mu$. This is due to the current trim algorithm in VAST when using free-wake, which will be changed in future. In hover no simulation was performed with VAST because with this setup the wing would be standing in still air thus would not generate a vortex at all, leaving the rotor in undisturbed air. The wing circulation strength depends on $\mu$. In the analytical model and in S4 the external vortex is modeled as space-fixed and this can be done at any advance ratio from zero to high speed and the vortex parameters are prescribed. Due to the differences in modelling the results obtained with the different methods can be compared only qualitatively. 


\section{Results}

The rigid straight-line vortex model is used by the analytic model, S4 and HOST, the deflected vortex model by S4 and the free-wake model in VAST includes the full mutuality of vortex-wake interaction. To eliminate the linear influence of $\lambda_{V 0}$ on the results the controls (in rad) required to retrim the rotor are divided by it: $\Delta \Theta / \lambda_{V 0}$ [8-12].

Very Small Advance Ratio $(\boldsymbol{\mu}=\mathbf{0 . 0 1})$. This is very close to hover with a shaft angle of $\alpha_{S}=-0.01^{\circ}$ and the slipstream angle $\chi$ is very small. The semi-empirical vortex deflection model can be applied, but it is still close enough to hover to be compared with hovering results using the rigid straight vortex assumption. Results from the analytical model, HOST, S4 with rigid straight-line and deflected vortex and are given in Fig. 2.
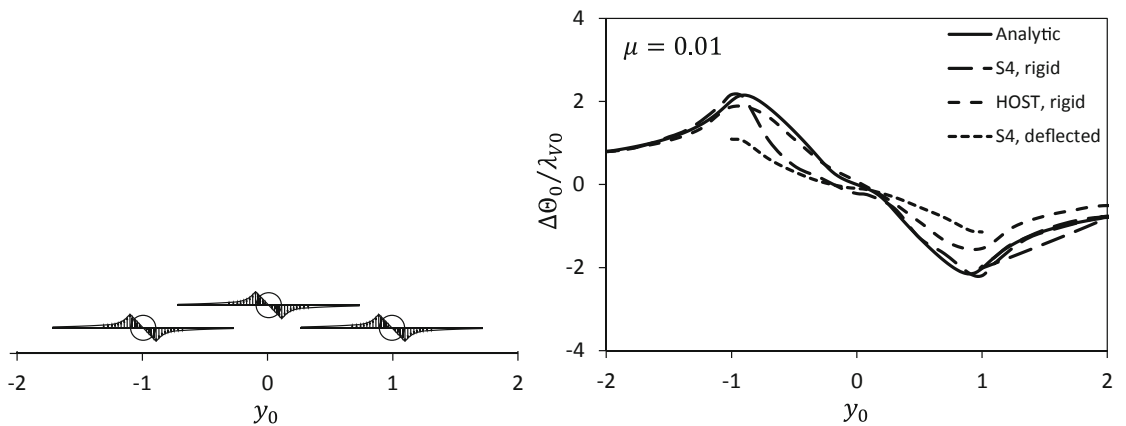

(a) Vortices placed at different lateral positions (b) Collective control angle

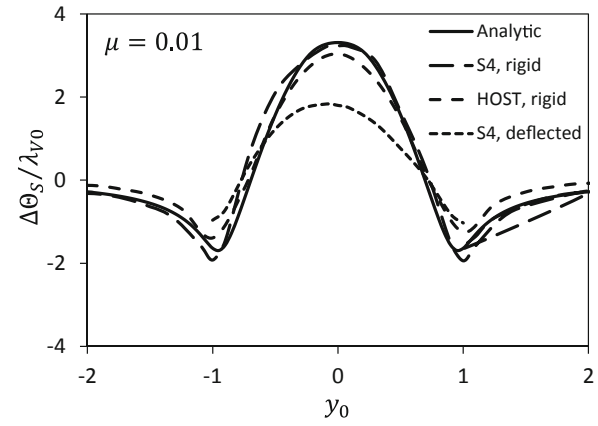

(c) Longitudinal cyclic control angle

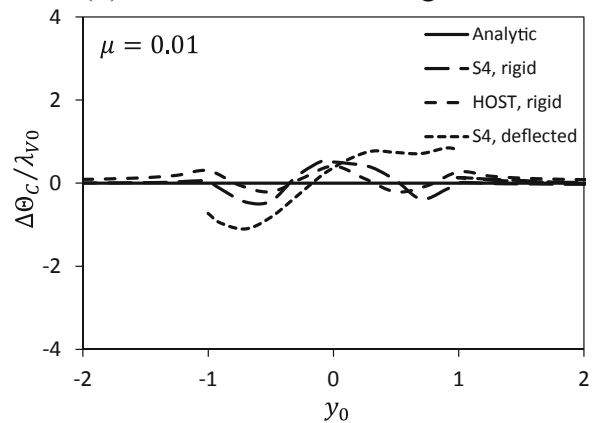

(d) Lateral cyclic control angle

Fig. 2. Vortex influence on rotor controls, $r_{c}=0.1, x_{0}=0$.

The collective control curves in Fig. 2 (b) for the analytical model and S4 using the rigid straight-line vortex are very close, while HOST predicts a little less magnitude, which is caused primarily by a coarser blade element resolution [10]. The vortex deflection model requires only about half of the control magnitude, because the vortex is straight and almost parallel to the rotor disk only in its front half while from the center on the vortex is steeply inclined downwards and therefore far away from the rear half of the rotor. Thus, its influence is mainly confined to only half of the disk and only 
half of the control magnitude is needed, compared to a rigid vortex [12]. The same observations can be made for the longitudinal control angles given in Fig. 2 (c). While the analytical model, S4 and HOST with rigid vortex assumptions generate results that are very close to each other, the deflected vortex model requires only about half of the control magnitude. The results obtained for the lateral control angle differ as shown in Fig. 2 (d). The analytical model requires no control input because of the longitudinal symmetry of induced velocities, the assumption of a central hinge with rigid blades, only flapping degree of freedom, and linear steady aerodynamics. S4 and HOST make use of elastic blades in flap, lag and torsion; therefore some coupling effects result in a minor amount of lateral control angle. The deflected vortex model requires more control angle input than the rigid straight-line vortex model because of the fore-aft asymmetry of vortex-induced velocities: a deflected vortex placed at $y_{0}=-1$ induces downwash in the front half of the disk as sketched in Fig. 2 (a), but not in the rear half of the disk due to its deflection at $x_{0}=0$. This longitudinal asymmetry of downwash requires a negative lateral cyclic control to compensate the loss of lift in the front side. In reverse, a vortex position at $y_{0}=+1$ induces upwash in the front half of the disk, but not so in the rear, and a positive lateral cyclic control angle is required to compensate this.

Variation of Advance Ratio. Results for advance ratios from $\mu=0$ to 0.4 are obtained here only with VAST and free-wake, Fig. 3. For the hovering case with $\mu=0$ no computation was performed with VAST, but obtained by physical consideration. At

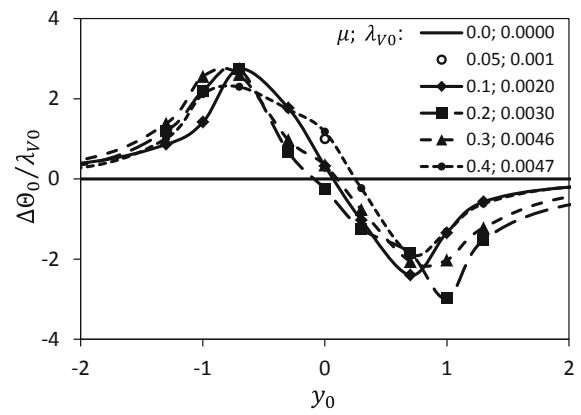

(a) Collective control angle

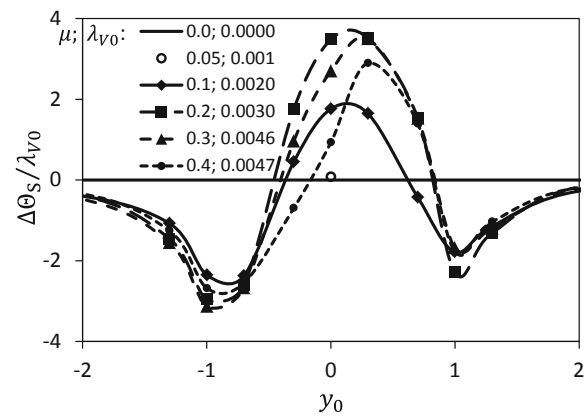

(b) Longitudinal cyclic control angle

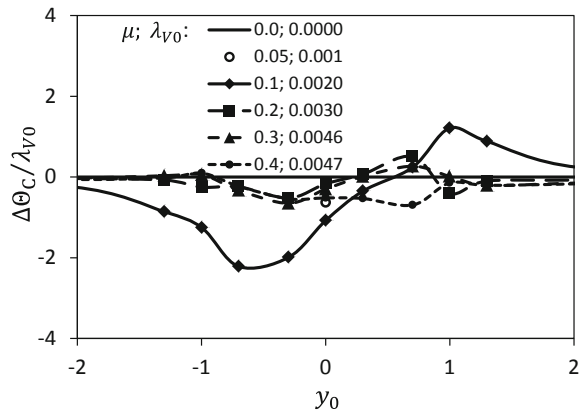

(c) Lateral cyclic control angle

Fig. 3. Influence of the advance ratio on rotor controls, free-wake results. 
$\mu=0.05$ the convergence of trim was very time consuming (due to the current trim algorithm in VAST when using free-wake that will be changed in future), therefore only one case with $y_{0}=0$ was computed (circle symbol).

The collective control angle shown in Fig. 3 (a) appears rather similar for every advance ratio. The lateral symmetry at small advance ratios gets progressively deformed with increasing advance ratio due to the lateral asymmetry of dynamic pressure acting on the rotor blades. The peak values become smaller with increasing advance ratio. More variation with advance ratio is found in the cyclic control angles, Fig. 3 (b) and (c). The longitudinal cyclic control angle shows less magnitude at the small advance ratio of $\mu=0.1$, largest values for $\mu=0.2$ and 0.3 , and smaller again at $\mu=0.4$, but with increasing asymmetry due to increasing difference in dynamic pressure on advancing and retreating side. Interesting is the larger amount of lateral control angle required at $\mu=0.1$, which in trend was predicted by S4 with the deflected vortex model, but for an even smaller advance ratio, see Fig. 2 (d).

The hypothesis that the controls should be zero in the hovering case is supported especially by the results for the longitudinal control angle, Fig. 3 (b), that has significantly smaller magnitudes for $\mu=0.1$, compared to $\mu=0.2$, especially visible at the vortex center position when $y_{0}=0$. A further reduction of $\mu$ to 0.05 (circle symbol) reduces the longitudinal cyclic control even more towards zero as assumed in hover. The lateral cyclic control angle shown in Fig. 3 (c) also has a large reduction when the advance ratio is reduced from $\mu=0.1$ to 0.05 .

In order to provide a feeling for the absolute values of control angles required to mitigate vortex effects, lets assume a vortex strength of $\Gamma_{V}=67 \mathrm{~m}^{2} / \mathrm{s}$, leading to $\lambda_{V 0}=0.01$ with Bo105 rotor radius and tip speed, using Eq. (1). Then, maximum values for vortex influence on controls are in the order of $\Delta \Theta / \lambda_{V 0} \approx 4$, thus $\Delta \Theta \approx 0.04 \mathrm{rad}$, or $2.3 \mathrm{deg}$. For larger vortex strength this becomes larger proportionally. At constant vortex strength $\Gamma_{V}$ and constant rotor tip speed $\Omega R$ the magnitude of $\Delta \Theta$ is proportional to $1 / R$, thus $\Delta \Theta$ is larger for smaller helicopters and smaller for larger helicopters than the Bo105.

\section{Visualization of Fixed-Wing and Rotor Wakes}

Small Advance Ratio $(\boldsymbol{\mu}=\mathbf{0 . 0 5})$. Due to the small freestream velocity the wing circulation also is very small in this case: $\lambda_{V 0}=0.001$. The rotor wake developing without the wing and its vortex is shown in Fig. 4 (a), denoted as "Baseline", and in (b) with the wing wake. This side view indicates hardly anything has changed for the rotor wake, but the wing vortex, due to upwash induced by the rotor, is convected upwards before reaching the rotor disk. When reaching the rotor it is sucked into the rotor slipstream away from its formerly horizontal path of convection. Therefore, the simple assumption of the deflected vortex model is essentially valid, provided that the location of deflection is properly computed.

Moderate Advance Ratio $(\boldsymbol{\mu}=\mathbf{0 . 1})$. The free-stream velocity still is relatively small and the wing vortex strength not large; in this case $\lambda_{V 0}=0.002$. As before, the isolated development of the rotor wake is shown in Fig. 5 (a). Compared to Fig. 4 the stronger 


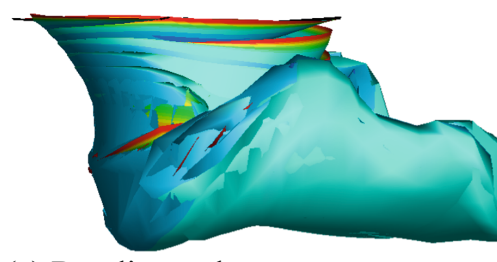

(a) Baseline wake, no vortex

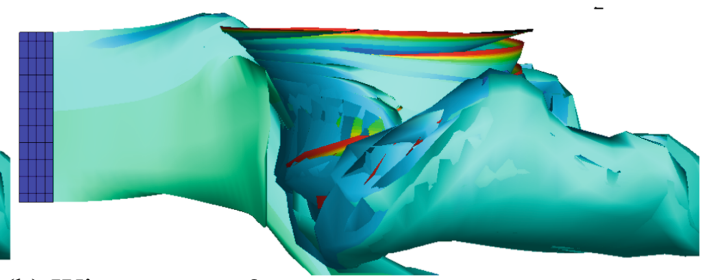

(b) Wing at $y=0$

Fig. 4. Vortex-wake interaction, $\boldsymbol{\mu}=0.05$, side view.

wing circulation results in a stronger deformation of the rotor wake. It can also be seen that the wing's vortex (the blue colored part of its wake) does not enter the rotor disk in its center, but rather more shifted to the right by about $10 \%$ of the rotor radius, although the wing is placed at $y_{0}=0$. The side view is given in Fig. 5 (c) for the combination of both where the wing is located at $y_{0}=0$. Compared to the small advance ratio of $\mu=0.05$ shown in Fig. 4 the rotor wake skew angle is already much larger and agrees with the momentum theory result given in Fig. 1 (c). The wing's wake is sucked into the rotor slipstream right behind the front of the rotor disk, and the entire wing wake is convected almost parallel to the rotor's slipstream. The bottom of the rotor wake is slightly lifted up relative to the Baseline wake, better seen in Fig. 5 (b), which is due to the sense of rotation of the wing vortex, inducing upwash on the rotor's retreating side and downwash on the other, as indicated by the red arrows.

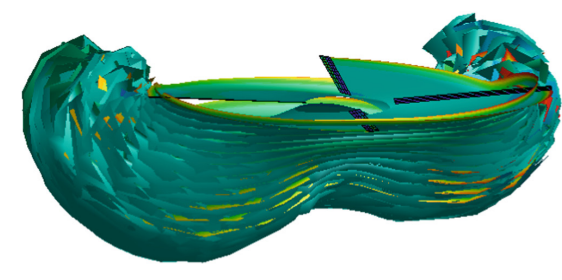

(a) Baseline wake, no vortex

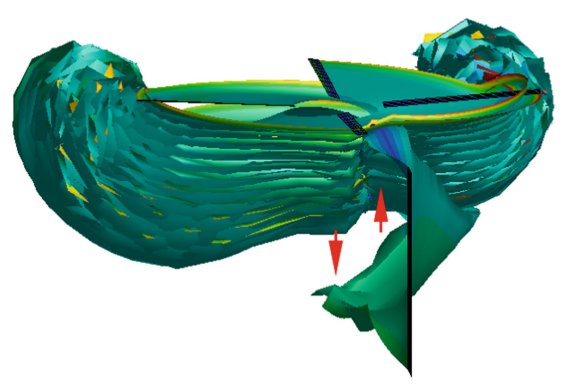

(b) Wing at $y_{0}=0$

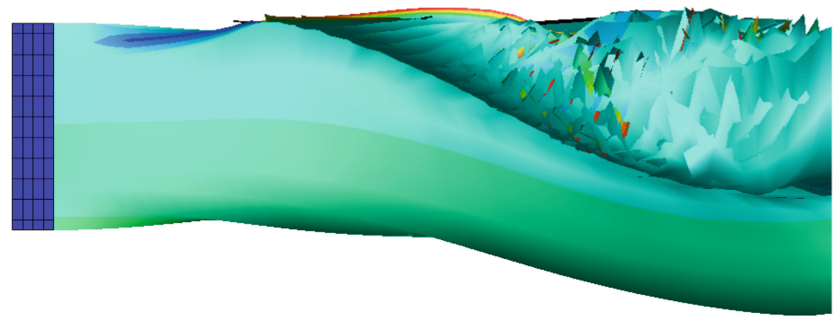

(c) Side view

Fig. 5. Vortex-wake interaction, $\boldsymbol{\mu}=\mathbf{0 . 1}$. 
High Advance Ratio $(\boldsymbol{\mu}=\mathbf{0 . 3})$. Now the wing's vortex circulation is $\lambda_{V 0}=0.0046$, the rotor wake becomes flatter and due to the high freestream velocity both wakes are quickly convected downstream. The combination of both wakes for a central wing position at $y_{0}=0$ is given in Fig. 6 (b). Compared to the smaller advance ratios shown before, the wing's wake is now more strongly deforming the rotor's wake in the area where it is immersed into it. The wing's wake is deformed by the rotor wake to a much lesser degree. The side view on the wing and rotor wake is given in Fig. 6 (c), where the rotor wake appears more as a system of rolled-up blade tip vortices well separated from each other, Fig. 6 (b), which remains as such when the wing's wake is affecting the rotor. The wake skew angle is very shallow in agreement with momentum theory, compare with Fig. 1 (c).

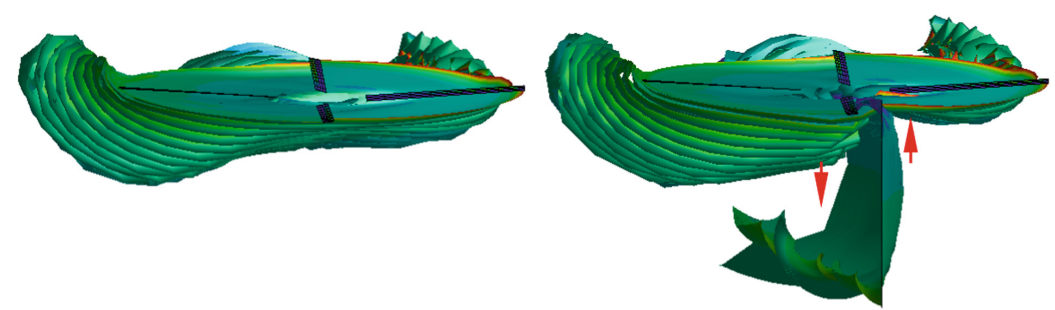

(a) Baseline wake, no vortex

(b) Wing at $y_{0}=0$

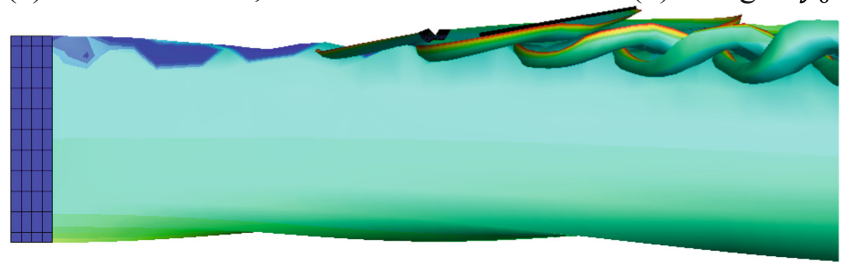

(c) Wing at $y_{0}=0$

Fig. 6. Vortex-wake interaction, $\boldsymbol{\mu}=\mathbf{0 . 3}$.

\section{Conclusions}

Three different modeling approaches of increasing complexity are applied to the problem of an external vortex interacting with a rotor and its wake and the results compared to each other, namely

- a rigid straight-line vortex model without any vortex-wake mutual interaction

- a deflected vortex model representing a unidirectional interaction from the rotor wake on the otherwise straight-line vortex

- a free-wake solution including all mutuality of the vortex acting on the wake and vice versa 
The main findings are summarized below.

1. For advance ratios $\mu \geq 0.2$ the simple methods using a rigid straight-line or deflected vortex are appropriate, because the vortex-wake mutual interactions are rather small within the rotor disk and develop mainly behind it, due to the large convection speed.

2. For smaller advance ratios around $\mu \approx 0.1$ the rigid straight-line vortex assumption is inadequate due to the strong wake deflection relative to the flight direction. The deflected vortex model generates reasonable results here, but the full mutuality of vortex-wake interactions provides better results, albeit requiring much higher computational effort.

3. For very small advance ratios down to hover also the deflected vortex model becomes questionable, because any vortex will be blown away by the rotor wake and the steady-state condition will be clean air. This can only be predicted by the free-wake approach, but at much higher computational effort compared to the comprehensive codes or the analytical method.

\section{References}

1. Johnson, W.: Rotorcraft Aeromechanics. Cambridge University Press, New York (2013)

2. Leishman, J.G.: Principles of Helicopter Aerodynamics. Cambridge University Press, New York (2000)

3. Sheridan, P.F., Smith, R.P.: Interactional aerodynamics - a new challenge to helicopter technology. J. Am. Helicopter Soc. 25(1), 3-21 (1980). https://doi.org/10.4050/JAHS.25.1.3

4. Mantay, W.R., Holbrook, G.T., Campbell, R.L., Tamaine, R.L.: Helicopter response of an airplane's trailing vortex. J. Aircr. 14(4), 357-363 (1977). https://doi.org/10.2514/3.58784

5. Azuma, A., Saito, S., Kawachi, K.: Response of a helicopter penetrating the tip vortices of a large airplane. VERTICA 11(1), 65-76 (1987)

6. Turner, G.P., Padfield, G.D., Harris, M.: Encounters with aircraft vortex wakes: the impact on helicopter handling qualities. J. Aircr. 39(5), 839-849 (2002). https://doi.org/10.2514/2. 3004

7. Bakker, R., et al.: Wind turbine wakes and helicopter operations (overview of the GARTEUR HC-AG23 activities). In: 44th European Rotorcraft Forum, Delft, Netherlands, 18-21 September 2018

8. van der Wall, B.G., van der Wall, L.B.: Analytical estimate of rotor controls required for a straight vortex disturbance rejection. J. Am. Helicopter Soc. 62(1), 015001-1-4 (2017). https://doi.org/10.4050/JAHS.62.015001

9. van der Wall, B.G.: Analytical estimate of rotor blade flapping caused by a straight vortex disturbance. J. Am. Helicopter Soc. 62(4), 045001-1-6 (2018). https://doi.org/10.4050/JAHS. 62.045001

10. van der Wall, B.G., Lehmann, P.H.: About the impact of wind turbine wake vortices on helicopter trim and rotor blade motion. In: 43rd European Rotorcraft Forum, Milano, Italy, 12-15 September 2017

11. van der Wall, B.G.: Estimate of rotor controls and blade flapping caused by an orthogonal vortex disturbance. J. Am. Helicopter Soc. 63(2), 025000-1-6 (2018). https://doi.org/10.4050/ JAHS.63.025001

12. van der Wall, B.G.: Effect of vortex deflection on vortex-rotor interaction. In: 45th European Rotorcraft Forum, Warsaw, Poland, 17-20 September 2019 
13. Whitehouse, G.R., Brown, R.E.: Modelling a helicopter rotor's response to wake encounters. Aeronaut. J. 108(1079), 15-26 (2004). https://doi.org/10.1017/S0001924000004954

14. Benoit, B., Dequin, A.-M., Kampa, K., von Grünhagen, W., Basset, P.-M., Gimonet, B.: HOST, a general helicopter simulation tool for Germany and France. In: 56th Annual Forum of the American Helicopter Society, Virginia Beach, VA, 2-4 May 2000

15. van der Wall, B.G., Lim, J.W., Smith, M., Jung, S.N., Bailly, J., Baeder, J.D., Boyd, D.D.: The HART II international workshop: an assessment of the state-of-the-art in comprehensive code prediction. CEAS Aeronaut. J. 4(3), 223-252 (2013). https://doi.org/10.1007/s13272013-0077-9

16. van der Wall, B.G., Roth, M.: Free-wake analysis on massively parallel computers and validation with HART test data. In: 53rd Annual Forum of the American Helicopter Society, Virginia Beach, VA, 29 April-1 May 1997 